\title{
PARRY ROMBERG SYNDROME
}

\author{
Alexis-Virgil Cochino ${ }^{1,2}$, Sigrid Covaci, ${ }^{1,2}$, Oana-Maria Farkas ${ }^{1}$ \\ ${ }^{1}$ Alessandrescu-Rusescu National Institute for Mother and Child Health, Bucharest \\ ${ }^{2}$ Carol Davila University of Medicine and Pharmacy, Bucharest
}

\begin{abstract}
Parry-Romberg syndrome, which is also known as progressive facial hemiatrophy, is a variant of localized sclero-
derma. This is a rare condition (OMIM \# 141300), characterized by a progressive but self-limited atrophy of the
skin and subcutaneous tissue on one side of the face. We present the case of a 9 year old boy with a typical
presentation of hemifacial atrophy, with Borrelia burgdorferi infection without any changes of the nervous system.
\end{abstract}
Keywords: Parry - Romberg syndrome, progressive facial hemiatrophy, scleroderma

\section{INTRODUCTION}

The localized sclerodermas (LS) are a group of diseases which damage mostly the skin and subdermal tissues and generally do not affect the internal organs (1). Linear scleroderma, the most common subtype in patients under 18 years old, is characterized by one or more linear streaks that may extend from the dermis, through subcutaneous tissue and muscle to the bone (2). When occuring on the face and head, they can take the form of either a "sabercut" - like lesion (en coup de sabre) or progressive hemifacial atrophy (also known as Parry-Romberg syndrome), the latest implying minimal epidermal changes, but marked dermal and subcutaneous atrophy (3). The etiology of Parry Romberg syndrome (PRS) is unknown. There are reports that suggest that the causes could be autoimmunity, drugs, local trauma and infection with Borrelia burgdorferi. When occuring on the face or forehead, the localized scleroderma onset is typically in the first or second year of life (4). Clinical manifestations of PRS include: facial hemiatrophy with progressive evolution, loss of the subcutaneous fat in the affected region, hemiatrophy of tongue, deviation of nose, angle of mouth and chin to the affected side, cutaneous changes like hyperpigmentation and atrophy. Parry Romberg syndrome may be associated with neurological symptoms, especially seizures and mi- graines, dental abnormalities and ocular manifestations. The diagnosis is based especially on the clinical features. PRS is usually a self-limiting condition, with an uncertain treatment and prognosis (4). There is no standardized regimen; generally, steroids are used along with immunosuppressors.

\section{CASE PRESENTATION}

In august 2013, a 9 - year old boy was referred to Paediatric Department of INSMC "AlessandrescuRusescu" for the diagnosis. The patient's medical and family history was insignificant. The disease onset was in February 2013, with erythematous violaceous areas and slightly edematous aspect of the infraorbital left zone, evolving with atrophicand pigmentation areas. In June 2013, a dermatological exam highlights "skin thickening on the left side of the face, atrophy of the left maxillary and zygomatic region and left side enophtalmy, resulting in facial asymmetry and faded folds on the left side of the face; thickened area and hyperpigmented areas". This clinical aspect suggested the diagnosis of progressive hemifacial atrophy (Parry Romberg Syndrome). Routine blood tests, antibody testing, Borrelia burgdorferi serology, skin biopsy and cerebral MRI were performed. Patient was positive for IgM Borellia burgdorferi, negative for antinuclear antibodies and rheumatoid factor. The skin biopsy 
showed perivascular and perianexial dermatitis, predominantly lobular panniculitis with lymphocytes and plasma cells, with thinned epidermis; abundant infiltrate, sometimes nodular, predominantly perivascular and perianexial, and discreet interstitial infiltrate; increased amount of mucin in reticular dermis and sclerosis of the deep reticular dermis; hyalinisation of the fat lobe. The cerebral MRI revealed a low position of the tonsils. The ophthalmic examination was normal.

In august 2013, the physical examination revealed a healthy boy with normal development and nutritional status, facial asymmetry with left facial atrophy and faded folds (Fig. 1), skin thickening, loss of subcutaneous fat in the left maxillary and zygomatic region, enophtalmy of the left eye, deviation of the nose, angle of mouth and chin to the left side (Fig. 1), atrophy of the left side of the tongue (Fig. 2), atrophy of buccinator and maseter muscles and prominent zygomatic arch on the left side, cutaneous hypoesthesia in the left side of the face. Vital signs, speech and hearing were normal, and no signs of mental instability were present. The blood tests were normal, excepting a mildly increased erythrocyte sedimentation rate (ESR) and positive antinuclear antibodies ( $2^{\text {nd }}$ determination). Rheumatoid factor and anti-double-stranded DNA antibodies were not present. Anticentromere, anti-Scl70 and other antibodies were not performed. Cerebral CT scan was normal, while CT scan of the face revealed facial asymmetry with the absence of subcutaneous tissue in the entire left malar region, reduced thickness of the upper lip muscles and the orbicularis

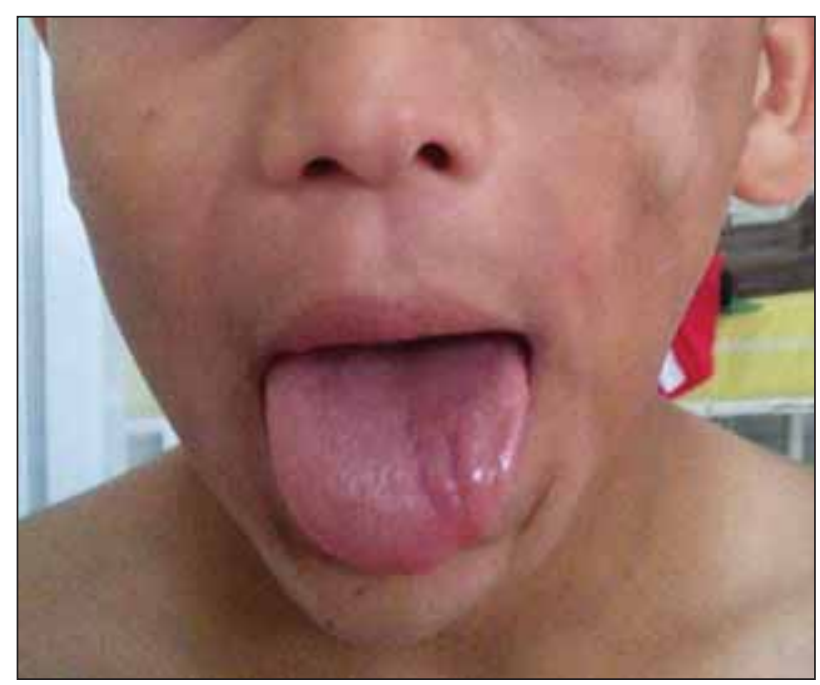

FIGURE 1. Facial asymmetry with left facial atrophy and faded folds; enophtalmy of the left eye, deviation of the nose, of the mouth angle and chin to the left side

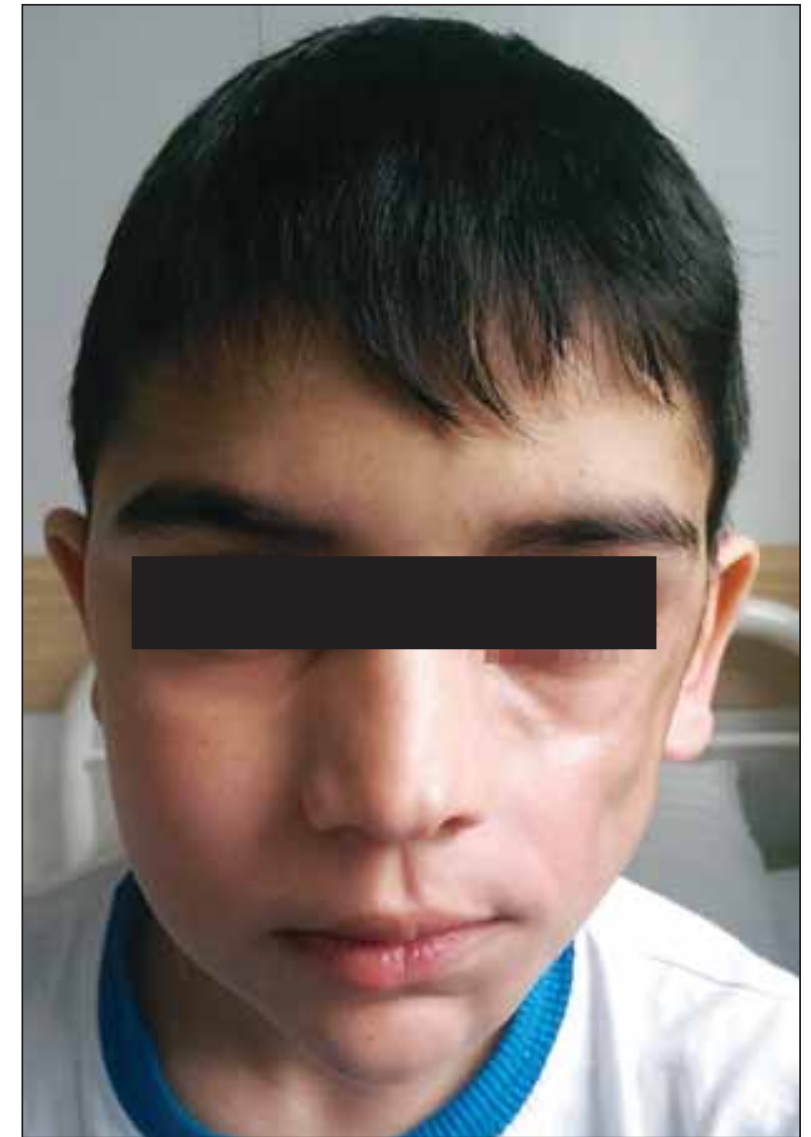

FIGURE 2. Left side of the tongue atrophy

lifter of the left side (compared to the right side); chronic inflammatory changes of the maxillary sinuses and right compartment of sphenoid sinus were also described. As such, the imagistic aspect was compatible with the diagnosis of Parry-Romberg syndrome (progressive hemifacial atrophy), which was based on the clinical and imagistic findings. Therapy was started with methylprednisolone $(30 \mathrm{mg} / \mathrm{kg} /$ day iv -3 days/month for 6 consecutive months) and methotrexate $10 \mathrm{mg} /$ week. After 5 months of treatment, the evolution was slowly favourable and the patient was switched to oral steroids (prednison $10 \mathrm{mg} /$ day). Further evolution was favourable with the reduction of skin induration. Three months later, prednison was tapered and ultimately stopped. Methotrexate was maintained for 12 months. Under this treatment, the evolution was favourable with important reduction of skin induration and mild recovery of the subcutaneous fatpad. At the time of this article's writing, the patient is being prepared for surgical reconstruction/ cosmetic correction.

\section{DISCUSSIONS}

A case of PRS associated with Borrelia burgdorferi infection, with no neurologic or ophtalmologic 
manifestations is presented. Although under debate as a direct relationship, timely association between PRS and Borrelia burgdorferi was first reported in 1985 (5). As such, and in the lack of clinical signs of active borreliosis, the patient was not treated with antitbiotics.

Despite the lack of consensus regarding the PRS's treament and in the view of a highly active and fast evolving disease form, the patient was treated with a combination of steroids (pulsed therapy subsequently followed by oral administration) and an immunosuppressor (i.e. metothrexat), with a good evolution regarding the disease activity control.

Similar to other localized sclerodermas, PRS evolves with a phase of activity, which is self-limited. The treatment aims at reducing tissue damage and loss of function and not at shortening the disease duration.

\section{CONCLUSION}

Parry Romberg syndrome, a form of localized scleroderma sometimes cited in association with Borrelia burgdorferi infection, needs prompt evaluation and treatment, in order to avoid permanent sequeallae, especially of the eye and nervous system. If needed, immunosuppressors should be used, tuning the intensity according to the disease level of activity. More research is needed in order to recommend a standardized regimen. Treatment is usually needed only during the disease's active phase, which in the presented case lasted a couple of years.

\section{REFERENCES}

1. Francesco Zulian, Ronald M. Laxerin in Textbook of pediatric rheumatology, 6 th Ed - Philadelphia, PA: Elsevier/Saunders, 2011

2. Francesco Zulian in Handbook of Systemic Autoimmune Diseases. Volume 6 Pediatrics in Systemic Autoimmune Diseases. Amsterdam, Elsevier, 2008

3. Ronald M. Laxer, Susanne M. Benseller in Kelley's Textbook of Rheumatology. Philadelphia, PA: Elsevier/ Saunders, 2013

4. Thomas J.A. Lehman. A clinician's guide to rheumatic disease in children. New York, Oxford University Press, 2009

5. Aberer E., Neumann R., Stanek G. Is localised scleroderma a Borrelia infection? [letter]. Lancet, 2 (1985) 